\title{
Detection of IL-6 by magnetic nanoparticles grown with the assistance of mid-infrared lighting
}

\author{
XIUFENG JIANG ${ }^{1,2}$, YE ZHANG ${ }^{1}$, XIAOFEI MIAO ${ }^{1}$, ZENGHUI LI $^{1}$, ZENGTAO BAO $^{1}$ and TONG WANG $^{1,2}$ \\ ${ }^{1}$ Department of General Surgery, Wuxi People's Hospital, Wuxi, Jiangsu 214023; \\ ${ }^{2}$ Nanjing Medical University, Nanjing, 210029, P.R. China
}

Received July 15, 2012; Accepted October 10, 2012

DOI: $10.3892 / \mathrm{mmr} .2012 .1136$

\begin{abstract}
Nanomedical systems have attracted considerable attention primarily due to suitability in applications for specific cell selection through biomolecular targeting and rare cell detection enhancement in a diverse, multicellular population. In the present study, magnetic nanoparticles were prepared for use in high accuracy cell sensing. Magnetic nanoparticle growth was assisted by mid-infrared lighting. By this mechanism, a narrow window, estimated to be $2 \%$, was achieved for the dimension distribution of grown nanoparticles. Combined with silicon nanowire (SiNW) transistors, a sensor with ultra high sensitivity for the detection of specific potential low abundance biomarkers has been achieved, which has been specifically used to detect interleukin-6 (IL-6) at extremely low concentrations. A novel biosensor with high sensitivity has been fabricated and utilized in the detection of IL-6 at $75 \mathrm{fM}$ to $50 \mathrm{pM}$. The system consists of an SiNW transistor and magnetic nanoparticles with even dimension distribution. The novel sensor system is suitable for quantifying IL-6 at low concentrations in protein samples.
\end{abstract}

\section{Introduction}

Nanomedical systems are becoming important methods for cell selection applications, including specific cell selection by biomolecular targeting and rare cell detection enhancement in diverse, multicellular populations $(1,2)$. Nanoparticles (3-6), microcantilevers $(7)$ and carbon nanotubes $(8,9)$ have been used to detect binding and unbinding of proteins in human serum. These systems have demonstrated high selectivity and femtomolar sensitivity compared with the enzyme-linked immunosorbent assay (ELISA).

Correspondence to: Dr Tong Wang, Department of General Surgery, Wuxi People's Hospital, 299 Qingyang Road, Wuxi, Jiangsu 214023, P.R. China

E-mail: aanti@163.com

Key words: nanoparticles, detection, interleukin-6, mid-infrared lighting
Interleukin-6 (IL-6) is a pleiotropic cytokine that is overexpressed in response to injury, inflammation and infection $(10,11)$. Under normal physiological conditions serum levels of IL-6 are low or undetectable. However, the production of IL-6 is regulated by several physiological factors, including diet, exercise and stress (12). The role of IL-6 in cancer is complex and includes autocrine and paracrine mechanisms. Numerous tumor cells from prostate, breast and colon cancer produce large amounts of IL-6 $(13,14)$. The use of IL-6 as an identification indicator for nanomedical systems is a widely accepted method.

Magnetic nanoparticles are commonly used in nanomedical systems and function as attractive materials in biomedicine due to altered magnetic behavior compared with bulk materials. For example, magnetic fine particles are currently being investigated for application in hyperthermia treatments, magnetic separation, drug delivery, tissue engineering and magnetic resonance imaging contrast enhancement (15-20). The most common magnetic nanoparticles utilized in biomedicine are iron oxide nanoparticles $\left(\mathrm{Fe}_{2} \mathrm{O}_{3}\right.$ and $\left.\mathrm{Fe}_{3} \mathrm{O}_{4}\right)$. However, the low saturation magnetization property of these nanoparticles has limited their application. In addition, other magnetic nanoparticles with higher magnetization levels, including FeCo, exhibit severe oxidation issues which are incompatible with the human body, also hindering bioapplication.

A feasible solution to these issues currently under investigation involves coating the magnetic nanoparticles with organic or inorganic materials to form core-shell nanostructures (16-20). In the present study, silica was adopted to coat iron-cobalt material, primarily since silica does not affect the optical properties of the nanoparticles due to its transparency.

Several synthetic methods have been used to synthesize FeCo nanoparticles, including thermal decomposition (16), chemical vapor condensation (17), arc discharge (18), sol-gel (19) and laser pyrolysis (20). In the present study, a novel method for growing ( $\mathrm{FeCo}) \mathrm{Si}$ core-shell structure is introduced. Mid-infrared lighting was applied during material growth, which has been demonstrated as an effective supplemental measure to obtain nanoparticles with narrow dimension distribution.

Following obtainment of the nanoparticles for biomarker detection, a silicon nanowire transistor (SiNW) was used. Prepared nanoparticles were attached to a nanowire. By observing transistor resistance, various indicator levels were 
measured. The concrete measurement method and results are included in the present study.

\section{Materials and methods}

Protein samples. IL-6 was purchased from Calbiochem (La Jolla, CA, USA). All the protein samples were used as received, without further purification and diluted in the assay buffer ( $1 \mathrm{mM}$ phosphate buffer solution containing $2 \mathrm{mM} \mathrm{KCl}$, $\mathrm{pH}$ 7.4) prior to sensing measurements. The IL-6 antibody was purchased from Invitrogen Life Technologies Corp. (Carlsbad, CA, USA).

ELISA. IL-6 levels in protein samples were determined using the Human IL-6 Chemiluminescence ELISA kit (Invitrogen Life Technologies Corp.).

Preparation of magnetic nanoparticles. Magnetic labels (magnetic nanoparticles) play a critical role in magnetic biosensing, as the size of magnetic labels determines the accuracy, particularly for small amounts of biomarkers.

Magnetic labels are commercially available in sizes ranging between $50 \mathrm{~nm}$ and $3 \mu \mathrm{m}$, however, these labels currently exhibit low diffusivity and poor binding selectivity.

Based on these current issues, an ideal magnetic nanoparticle acting as label should have the following properties (20): i) high-moment magnetic nanoparticles; ii) coated with silicon or gold to be biocompatible; iii) small size; and iv) distribution window of nanoparticles should be as narrow as possible.

The system shown in Fig. 1 was used in the present study for nanoparticle preparation. High-moment multifunctional nanoparticles are synthesized directly from the gas phase by using a physical vapor nanoparticle-deposition technique.

The target in Fig. 1 is $\mathrm{Fe}_{60} \mathrm{Co}_{40}$. As shown in Fig. 1, the heater and radio frequency (RF) source $(13.56 \mathrm{MHz})$ were simultaneously applied to vaporize the target. The continual sputtering gas was applied to ensure the vaporized target atom flowed in the same direction. Electrodes were also applied to speed up the mobility of the vaporized atoms. Argon is used as either sputtering gas or cooling media at the same time. When the supersaturation condition is reached, the vaporized target atoms form a nucleus and continue to grow into nanoparticles. By controlling system conditions, including target composition, target setup, plasma density and gas flow, magnetic nanoparticles are formed with controlled size, size distribution, composition, phase and structure. The base pressure of the system was $5 \times 10^{-8}$ Torr. An additional gas tube, for silicon atoms, does not appear in Fig. 1, however, the silicon is coated following completion of the nanoparticle nuclei.

Preparation of sensor system. A sensor is required to combine with the prepared magnetic nanoparticles. The measuring principle involves nanoparticles acting as labels, which combine with antibodies. When the specific biomarker appears, labels with attached antibodies capture biomarkers. Therefore, another sensor is required to integrate with nanoparticles and indicate the variations during measurement.

When the sensor is exposed to the biomarker, it combines with the biomarker through the antibody. Prior to this, biomarkers are combined with nanoparticle labels and there-

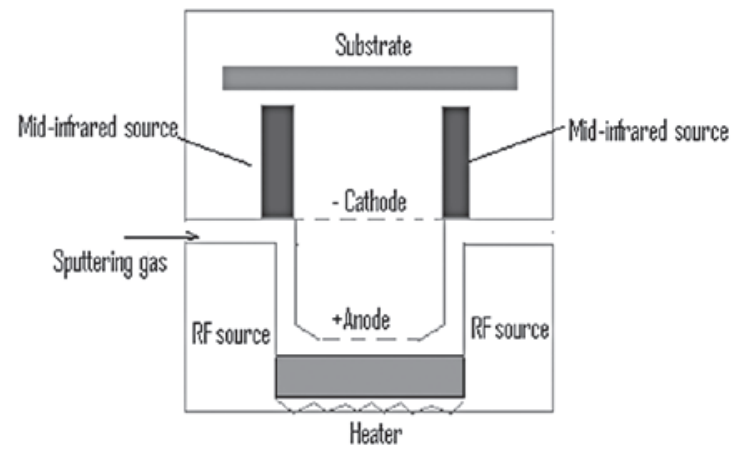

Figure 1. Schematic image of instrument used to fabricate the nanoparticles. $\mathrm{RF}$, radio frequency.

A
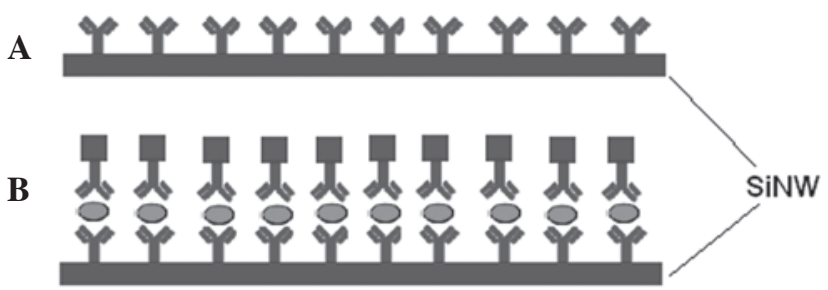

Capture antibody

High moment FeCo magnetic nanoparticle

Biomarker

Magnetic nanoparticles modified detection antibody

Figure 2. A novel biomarker detection scheme using the SiNW transistor as a biosensor and high moment FeCo magnetic nanoparticles is shown. (A) SiNW with antibody; (B) SiNW with biomarker captured by magnetic nanoparticles. SiNW, silicon nanowire.

fore the sensor is combined with nanoparticles in this way. The properties of the sensor vary following combination with the biomarker and therefore the sensor is used to observe the presence and levels of the biomarker.

A suitable sensor for the nanoparticles must be sensitive and measureable. In the present study a SiNW acts as sensor. The principle is shown in Fig. 2. Fig. 2A demonstrates SiNW prepared with antibody attached. Fig. 2B demonstrates high moment (FeCo)Si nanoparticles bound with SiNW through a biomarker.

Sample detection. To avoid the effects of atmosphere and light, all measurements were performed in a vacuum and in a dark environment. A passivator layer is likely to be required for future biomedical applications.

\section{Results}

(FeCo)Si nanoparticles. Fig. 3 shows the TEM image of particles, revealing that the structure is a core-shell, with cubic shape core. Shell thickness was estimated to be $3 \mathrm{~nm}$. Fig. 4 demonstrates selected area electron diffraction patterns of core-shell structured nanoparticles. Lattice length was measured and indicated a iron-cobalt bcc structure.

A comparison was made to demonstrate the function of mid-infrared lighting in particle size distribution. Two groups of samples were prepared, one assisted with mid-infrared 


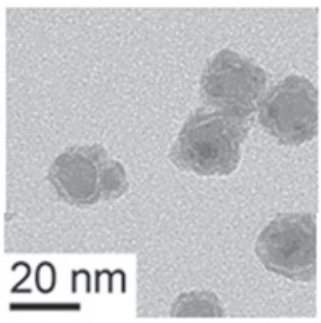

Figure 3. TEM image for the particles, the inset length is $20 \mathrm{~nm}$.

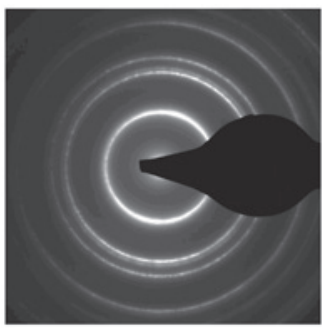

Figure 4. Selected area electron diffraction patterns of core-shell structured nanoparticles.

A

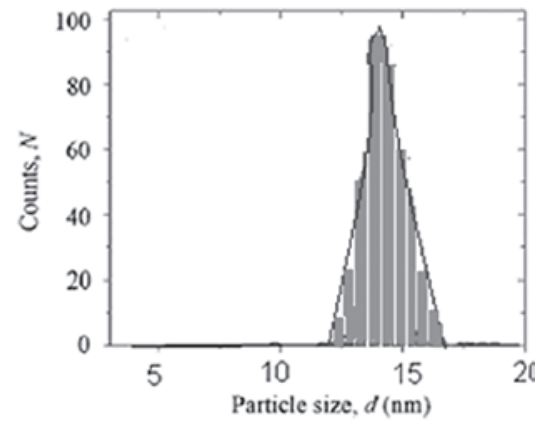

B

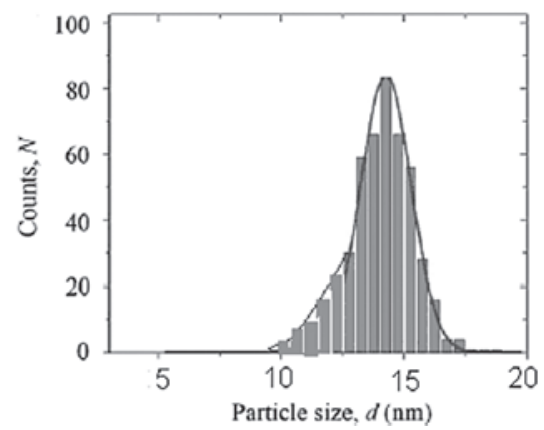

Figure 5. Size distribution of ( $\mathrm{FeCo}$ ) silicon nanoparticles. (A) Sample with infrared lighting, mean size, $14 \mathrm{~nm}$ and $2 \%$ distribution. Line exhibits normal distribution. (B) Sample without infrared lighting, mean size, $14 \mathrm{~nm}$ and 10\% distribution.

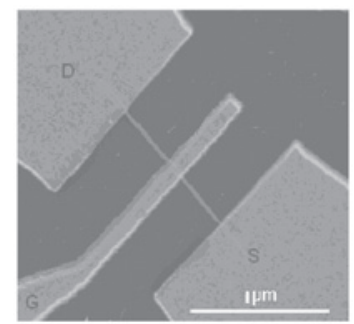

Figure 6. SEM photograph of silicon nanowire transistor.

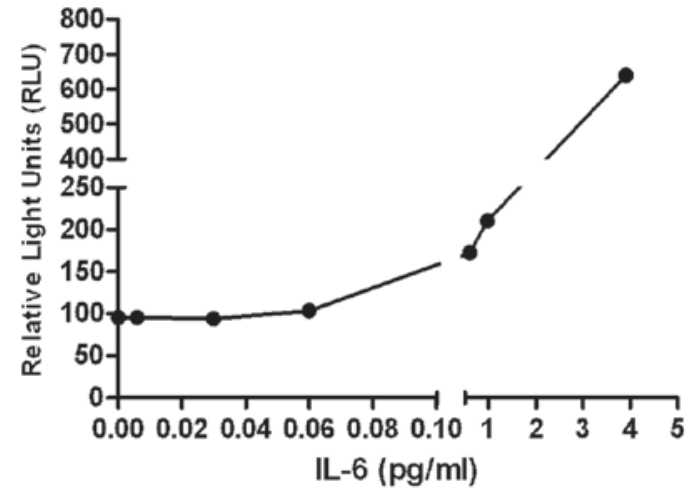

Figure 7. ELISA with different concentrations of IL-6. IL-6, interleukin-6; ELISA, enzyme-linked immunosorbent assay.

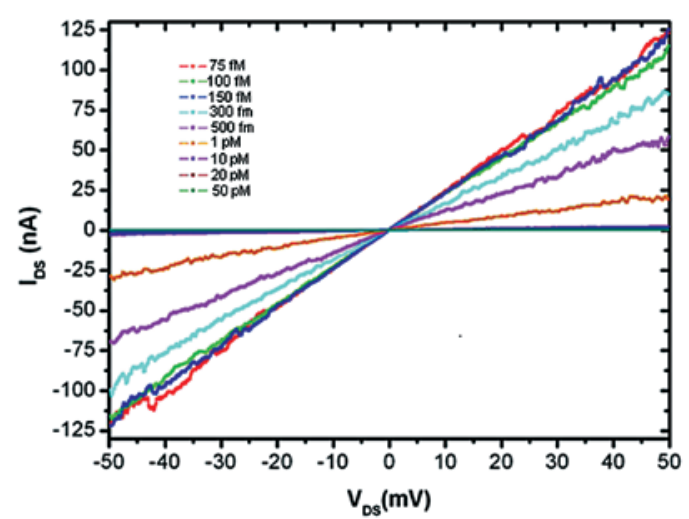

Figure 8. Current-voltage curves of the transistor at different doses, $75 \mathrm{fM}-50 \mathrm{pM}$.

lighting and the other without lighting. Fig. 5 demonstrates the size distribution of the samples.

Fig. 5A demonstrates the size distribution of nanoparticles with infrared lighting. A mean size of $14 \mathrm{~nm}$ was observed and the distribution window was $2 \%$. The line exhibited a normal distribution. Fig. 5B demonstrates the size distribution of nanoparticles without infrared lighting. The mean size was the same as nanoparticles with infrared lighting, however, the distribution window was $10 \%$. Therefore, infrared lighting has an effect on the size distribution of magnetic samples (Fig. 5). Since size distribution of particles has an effect on the sensitivity of biosensor, infrared lighting is utilized to increase sensitivity. To date, this is the narrowest window for nanoparticles observed.

Sensor system. The preparation of SiNW is not included in this study due to length limitations. A popular growth method in MBE (Molecular Beam Epitaxy) was used for preparation (21), assisted by plasma generated by the RF source inside the MBE system.

The prepared SiNW was $15 \mathrm{~nm}$ in diameter and exhibited an ideal doping profile along its axis. Fig. 6 shows an SEM image, in which drain, source and gate are annotated.

Comparison of IL-6 detection by SiNW transistor biosensor and ELISA. To demonstrate the practical application of high 
sensitivity SiNW transistor biosensors for biomarker detection, detection of human IL-6 was performed, following principles used in ELISA. It was observed that at IL-6 concentrations $<1 \mathrm{pg} / \mathrm{ml}$, ELISA did not generate a sensitive result (Fig. 7).

Based on the data in Fig. 8, it is revealed that the novel method described in the present study detects IL- 6 between $75 \mathrm{fM}$ and 50pM, the most sensitive method reported thus far. When the dose is fixed, the I-V curve of the transistor appears lineally, indicative of stable nanoparticle attachment to the nanowire surface, unaffected by electron flow.

\section{Discussion}

Traditional technologies for protein detection are enzymelinked immunosorbent assays (ELISA), western blotting, immunohistochemistry and DNA-based genomics. In the present study, a novel high sensitivity sensor, composed of a SiNW transistor combined with high-moment magnetic nanoparticles, was proposed and demonstrated.

By employing our novel SiNW transistor sensor with the high-homogenous (FeCo)Si nanoparticles, the sensor was employed to perform accurate and rapid quantification of IL-6, a low-abundance protein and potential cancer biomarker, in unprocessed human serum.

The SiNW transistor sensor system is 20 times more sensitive than that of the IL-6 ELISA and may be suitable for quantification of low abundance biomarkers in biological samples. The SiNW transistor sensor system binds with the same antibody utilized in the ELISA and therefore may function as an important supplement to the current commercial ELISA system. Though the SiNW transistor sensor system has a high sensitivity, the validation of the specificity of the SiNW transistor sensor system in our research is insufficient. Additionally, we only detected the different levels of IL-6 in protein samples, not in patient serum samples. In our future research we will investigate the method for using the SiNW transistor sensor system to detect IL-6 in human serum protein, which has more complex conditions.

In conclusion, the SiNW transistor sensor system is suitable for quantifying very small amounts of IL-6 in protein samples. Additionally, it may supplement the current commercial ELISA system.

\section{Acknowledgements}

This study was supported by the National Natural Science Foundation of China (no. 81141075/H1819) and Medical Technology Major Projects of Wuxi Hospital Center (YGZX1105).

\section{References}

1. Macy EM, Hayes TE and Tracy RP: Variability in the measurement of C-reactive protein in healthy subjects: implications for reference intervals and epidemiological applications. Clin Chem 43: 52-58, 1997.

2. Engvall E and Perlmann P: Enzyme-linked immunosorbent assay (ELISA). Quantitative assay of immunoglobulin G. Immunochemistry 8: 871-874, 1971

3. Alivisatos P: The use of nanocrystals in biological detection. Nat Biotechnol 22: 47-52, 2004.

4. Gao X, Cui Y, Levenson RM, et al: In vivo cancer targeting and imaging with semiconductor quantum dots. Nat Biotechnol 22: 969-976, 2004

5. Soukka T, Paukkunen J, Harma H, Lonnberg S, Lindroos H and Lovgren T: Supersensitive time-resolved immunofluorometric assay of free prostate-specific antigen with nanoparticle label technology. Clin Chem 47: 1269-1278, 2001.

6. Nam JM, Thaxton CS and Mirkin CA: Nanoparticle-based bio-bar codes for the ultrasensitive detection of proteins. Science 301: 1884-1886, 2003.

7. Wu G, Datar RH, Hansen KM, et al: Bioassay of prostate-specific antigen (PSA) using microcantilevers. Nat Biotechnol 19: 856-860, 2001

8. Chen RJ, Bangsaruntip S, Drouvalakis KA, et al: Noncovalent functionalization of carbon nanotubes for highly specific electronic biosensors. Proc Natl Acad Sci USA 100: 4984-4989, 2003.

9. Chen RJ, Choi HC, Bangsaruntip S, et al: An investigation of the mechanisms of electronic sensing of protein adsorption on carbon nanotube devices. J Am Chem Soc 126: 1563-1568, 2004.

10. Bosch FX, Ribes J, Diaz M and Cleries R: Primary liver cancer: worldwide incidence and trends. Gastroenterology 127: S5-S16, 2004.

11. Scheller J and Rose-John S: Interleukin-6 and its receptor: from bench to bedside. Med Microbiol Immunol 195: 173-183, 2006.

12. Pedersen BK and Febbraio MA: Muscle as an endocrine organ: focus on muscle-derived interleukin-6. Physiol Rev 88: 1379-1406, 2008.

13. Mora LB, Buettner R, Seigne J, et al: Constitutive activation of Stat 3 in human prostate tumors and cell lines: direct inhibition of Stat 3 signaling induces apoptosis of prostate cancer cells. Cancer Res 62: 6659-6666, 2002.

14. Bromberg JF, Wrzeszczynska MH, Devgan G, et al: Stat3 as an oncogene. Cell 98: 295-303, 1999.

15. Swift DC, Gammel JT and Clegg SM: Treatment of compounds and alloys in radiation hydrodynamics simulations of ablative laser loading. Phys Rev E Stat Nonlin Soft Matter Phys 69: 056401, 2004.

16. Lee GH, Huh SH, Jeong JW, et al: Structural and magnetic properties of bimetallic FeCo nanoclusters. Korean Phys Soc 42: 367-370, 2003.

17. Wang ZH, Choi CJ, Kim BK, et al: Microstructure and magnetic property of $\mathrm{Fe}-\mathrm{Co}$ nanoparticles prepared by chemical vapor condensation process. J Alloys Compounds 351: 319-323, 2005.

18. Dong XL, Zhang ZD, Jin SR and Kim BK: Characterization and magnetic properties of Fe-Co ultrafine particles. J Magn Magn Mater 210: 143-144, 2000.

19. Basu S and Chakravorty D: Optical properties of nanocomposites with iron core-iron oxide shell structure. J Non Cryst Solids 352: 380-385, 2006.

20. Ong PL, Mahmood S, Zhang T, et al: Synthesis of cobalt ferrite nanoparticles by pulsed laser deposition in a diffusion cloud chamber. Surf Sci 254: 1909-1914, 2008.

21. Yanfeng $J$ and Yamin Z: Influence of gold particle size on melting temperature of VLS grown silicon nanowire. J Semicond 31: 012002, 2010. 